\title{
Reduced-gliotoxin induces ROS-mediated anoikis in human colorectal cancer cells
}

\author{
JUNXIONG CHEN ${ }^{1-3^{*}}$, QIONG LOU ${ }^{1-3^{*}}$, LU HE$^{1,2}$, CHUANGYU WEN $^{1,2}$, MENGMENG LIN $^{1,2}$, \\ ZEFENG ZHU ${ }^{1,2}$, FANG WANG ${ }^{1,2}$, LANLAN HUANG ${ }^{1,2}$, WENJIAN LAN ${ }^{4}$, \\ AIKICHI IWAMOTO ${ }^{5,7}$, XIANGLING YANG ${ }^{1,2}$ and HUANLIANG LIU ${ }^{1-3,6}$ \\ ${ }^{1}$ Guangdong Institute of Gastroenterology and the Sixth Affiliated Hospital, Sun Yat-sen University; \\ ${ }^{2}$ Guangdong Provincial Key Laboratory of Colorectal and Pelvic Floor Diseases, Guangzhou; \\ ${ }^{3}$ Department of Clinical Laboratory, The Sixth Affiliated Hospital, Sun Yat-sen University, Guangzhou, \\ Guangdong 510655; ${ }^{4}$ School of Pharmaceutical Sciences, Sun Yat-sen University, Guangzhou, Guangdong 510006, \\ P.R. China; ${ }^{5}$ Advanced Clinical Research Center, Institute of Medical Science, University of Tokyo, Tokyo, Japan; \\ ${ }^{6}$ Institute of Human Virology and Key Laboratory of Tropical Disease Control of the Ministry of Education, \\ Zhongshan School of Medicine, Sun Yat-sen University, Guangzhou, Guangdong 510080, P.R. China
}

Received June 27, 2017; Accepted January 30, 2018

DOI: 10.3892/ijo.2018.4264

\begin{abstract}
Reduced-gliotoxin is a small molecule derived from the secondary metabolites of marine fungi; compared to other gliotoxin analogues, it exhibits potent anticancer effects. However, the molecular basis of the death of colorectal cancer (CRC) cells induced by reduced-gliotoxin is unclear. Thus, the aim of this study was to investigate the potency of reducedgliotoxin against CRC cells and to elucidate the underlying mechanisms. Cell morphology, flow cytometric analysis and western bolt analysis were performed to examine the functions and mechanisms of cell death induced by reduced-gliotoxin. Our findings demonstrated that reduced-gliotoxin triggered rapid cell detachment and induced anoikis in CRC cells. Mechanistically, our data indicated that the anoikis induced by reduced-gliotoxin was associated with the disruption of
\end{abstract}

Correspondence to: Dr Xiangling Yang or Dr Huanliang Liu, Guangdong Institute of Gastroenterology and the Sixth Affiliated Hospital, Sun Yat-sen University, 26 Yuancun Er Heng Road, Guangzhou, Guangdong 510655, P.R. China

E-mail: yangx128@mail.sysu.edu.cn

E-mail: liuhuanl@mail.sysu.edu.cn

Present address: ${ }^{7}$ Japan Agency for Medical Research and Development (AMED), 1-7-1 Otemachi, Chiyoda-ku, Tokyo 100-0004, Japan

\section{${ }^{*}$ Contributed equally}

Abbreviations: ROS, reactive oxygen species; NAC, $\mathrm{N}$-acetylcysteine; MMP, mitochondrial membrane potential; ECM, extracellular matrix;

Key words: reduced-gliotoxin, colorectal cancer, anoikis, reactive oxygen species integrin-associated cell detachment and multiple signaling pathways. Furthermore, reduced-gliotoxin induced the excessive production of reactive oxygen species (ROS) and the disruption of mitochondrial membrane potential (MMP), resulting in the activation of both endogenous and exogenous apoptotic pathways and eventually, in the apoptosis of CRC cells. The blockage of ROS generation with $\mathrm{N}$-acetylcysteine (NAC) attenuated the anoikis induced by reduced-gliotoxin. Taken together, these results suggest that reduced-gliotoxin may prove to be a potential candidate in the treatment of CRC.

\section{Introduction}

Colorectal cancer (CRC) is one of the most commonly diagnosed types of cancer in both males and females worldwide $(1,2)$. While the surgical resection of localized disease may be curative, mortality is primarily caused by metastatic progression, even with therapeutic regimens and the use of targeted therapies $(3,4)$. Anoikis is an inherent cellular mechanism that 'cleans out' the detached epithelial cells in order to maintain tissue homeostasis and development (5-7). Cancer cells which survive during detachment from the extracellular matrix (ECM) and acquire metastatic potential have developed mechanisms with which resist anoikis $(8,9)$. The breakdown of anoikis contributes to the development of many types of cancer, predominantly mammary and colon cancers $(10,11)$. Therefore, enhancing the response of cancer cells to anoikis is considered to be an effective strategy for the treatment of metastatic colorectal cancer.

Marine fungi are an important source of structurally unique and biologically active natural products $(12,13)$. In recent years, research has been conducted on the metabolites of marine fungi and obtained the optimized cultivation parameters with which to isolate and identify a series of novel and/or bioactive metabolites (14-17). Gliotoxin and its analogues, the diketopiperazines of various fungal species, 
e.g.,Aspergrillus fumigatus, Eurotium chevalieri, Trichoderma virens, Neosartorya pseudofischeri, and some Prenicillium and Acremonium species, are known to be immunosuppressive agents (18) and have also been reported to have anticancer properties (19-23). Furthermore, reduced-gliotoxin has previously been shown to possess the most potent cytotoxic effect compared to other analogues in CRC cells (14). However, the detailed effects and molecular mechanisms responsible for the death of CRC cells induced by reduced-gliotoxin are unclear.

In this study, we examined the potency and of reducedgliotoxin against CRC cells and also aimed to elucidate the underlying mechanisms. We demonstrated that reducedgliotoxin triggered rapid cell detachment and induced the apoptosis of CRC cells. Mechanistically, our data indicated that the anoikis induced by reduced-gliotoxin was associated with the dysregulation of multiple signaling pathways and the disruption of integrin-associated detachment. Furthermore, excessive reactive oxygen species (ROS) production was induced by reduced-gliotoxin, resulting in the activation of both endogenous and exogenous apoptotic pathways, and ultimately culminating in the apoptosis of CRC cells. The blockade of ROS generation with $\mathrm{N}$-acetylcysteine (NAC) attenuated the activation of several protein kinase signaling pathways and cell apoptosis that was induced by reduced-gliotoxin. Taken together, our findings suggest that reduced-gliotoxin may prove be a potential candidate for the treatment of CRC.

\section{Materials and methods}

Reagents details and use. Reduced-gliotoxin was isolated from the secondary metabolites of the marine fungus, Neosartorya pseudofischeri, following the procedure described previously (14). The purity of reduced-gliotoxin was $>98 \%$ as determined by high-performance liquid chromatography (HPLC). Reduced-gliotoxin was dissolved in dimethyl sulfoxide (DMSO) to a $50 \mathrm{mM}$ stock solution and stored in a dark area at $-20^{\circ} \mathrm{C}$. LY294002, MK-2206 and Z-IETD-FMK (Selleck Chemicals, Houston, TX, USA) were dissolved in DMSO to a $50 \mathrm{mM}, 10 \mathrm{mM}, 50 \mathrm{mM}$ solution respectively, and stored at $-20^{\circ} \mathrm{C}$. The cells were pre-treated with a concentration gradient of LY294002 (up to $50 \mu \mathrm{M}$ ) MK-2206 (up to $5 \mu \mathrm{M}$ ) or Z-IETD-FMK (up to $50 \mu \mathrm{M}$ ) for $2 \mathrm{~h}$ and co-treated with reduced-gliotoxin for $24 \mathrm{~h}$. NAC (Cat. no. 1009005) and diphenyleneiodonium chloride (DPI Cat. no. D2926) (Sigma-Aldrich, St. Louis, MO, USA) was dissolved in DMSO to a $5 \mathrm{mM}$ solution. The cells were pre-treated with a concentration gradient of NAC (up to $5 \mathrm{mM}$ ) or DPI (up to $20 \mathrm{mM}$ ) for $2 \mathrm{~h}$ and followed by co-treatment with reduced-gliotoxin for $24 \mathrm{~h}$. Rabbit polyclonal anti-PARP (Cat. no. 9542), mouse monoclonal anti-caspase-8 (Cat. no. 9746), rabbit monoclonal anti-caspase-9 (Cat. no. 9502), rabbit monoclonal anti-Akt (Cat. no. 4691), rabbit monoclonal anti-phosphor-Akt (Ser473) (Cat. no. 4060), rabbit monoclonal anti-integrin $\beta 4$ (Cat. no. 14803), rabbit monoclonal anti-integrin $\beta 1$ (Cat. no. 9699), rabbit monoclonal anti-PUMA (Cat. no. 12450), rabbit monoclonal anti-MCL1 (Cat. no. 5453), rabbit monoclonal anti caspase-3 (Cat. no. 9665), rabbit monoclonal anti-phosphor- $\beta$-catenin (Thr41/Ser45) (Cat. no. 9565) and rabbit monoclonal anti-cleaved caspase-3 (Cat. no. 9661) antibodies were purchased from Cell Signaling Technology (Cell Signaling Technology, Beverly, MA, USA).
Rabbit monoclonal anti-glycogen synthase kinase (GSK)-3 $\beta$ (Cat. no. ab32391), rabbit monoclonal anti-phosphor-GSK-3 $\beta$ (Ser9) (Cat. no. ab75814), rabbit monoclonal anti- $\beta$-catenin (Cat. no. ab32572), and rabbit monoclonal anti-cleaved caspase-9 (Cat. no. ab2324) antibodies were purchased from Abcam (Abcam, Cambridge, MA, USA). Rabbit polyclonal anti-GAPDH (Cat. no. 10494-1-AP), and mouse monoclonal anti-ACTB (Cat. no. 60008-1-Ig) antibodies were purchased from Proteintech Group (Proteintech Group, Chicago, IL, USA).

Colorectal cancer cell lines and cell culture. The colorectal cancer cell lines, HCT116 and HT-29, were purchased from the Culture Collection of Chinese Academy of Science (Chinese Academy of Science, Shanghai, China). The cells were cultured in RPMI-1640 medium (\#11875500; Gibco/Thermo Fisher Scientific, Waltham, MA USA) containing 10\% fetal bovine serum (\#FBS-22A; Capricorn Scientific, Ebsdorfergrund, Germany) in a humidified atmosphere of $5 \% \mathrm{CO}_{2}$ at $37^{\circ} \mathrm{C}$.

Apoptosis assay. The cell apoptotic rate was determined by flow cytometry using an Annexin V-FITC/PI dual staining kit (Nanjing KeyGen Biotech, Nanjing, Jiangsu, China), according to the manufacturer's instructions. Briefly, the CRC cells were seeded in 6-well tissue culture plates, and were then treated with various concentrations for the indicated periods of time. Cells present in the supernatant cells were collected by centrifugation at $626 \mathrm{x}$ g for $10 \mathrm{~min}$. The cells were counted $\left(5 \times 10^{5}\right)$ and washed with PBS twice. The cells were then re-suspended in working solution (100 $\mu \mathrm{l}$ binding buffer with $5 \mu \mathrm{l}$ Annexin V-FITC and $5 \mu \mathrm{l}$ PI staining solution) for $10 \mathrm{~min}$ at room temperature in the dark. Subsequently, $400 \mu \mathrm{l}$ binding buffer were added immediately prior to analysis using a flow cytometer (BD Biosciences, San Jose, CA, USA). The resulting data were analyzed using BD FACSDiva software, version 6.1.3 (BD Biosciences).

Western blot analysis. Western blot analysis was performed as previously described (24) with minor modifications. Briefly, total cell lysates were prepared in lysis buffer containing both protease and phosphatase inhibitors (KeyGEN Biotech). Protein concentrations were measured using a Bio-Rad assay kit (Bio-Rad, Hercules, CA, USA). Total proteins were separated on SDS-PAGE gel and transferred onto PVDF membranes (Bio-Rad), and the membranes were then blocked with 5\% skimmed milk (BD Biosciences) in Tris-buffered saline with Tween-20 (TBS-T) and incubated with primary antibodies diluted in primary antibody solution I (Toyobo, Tokyo, Japan) overnight at $4^{\circ} \mathrm{C}$. Anti-PARP, caspase-8, caspase-9, phosphor-Akt (Ser473), integrin $\beta 4$, integrin $\beta 1$, PUMA, MCL1, caspase-3 phosphor- $\beta$-catenin (Thr41/Ser45) cleaved caspase-3, cleaved caspase- 9 antibodies were diluted at 1:1,000. Anti-Akt, GSK-3 $\beta$, phosphor-GSK-3 $\beta$ (Ser9) and $\beta$-catenin antibodies were diluted at 1:2,000. Anti-GAPDH and ACTB antibodies were diluted at 1:5,000. The following day, the membranes were washed and incubated with HRP-conjugated goat anti-rabbit IgG $(\mathrm{H}+\mathrm{L})$ (Cat. no. A16096; Invitrogen/Thermo Fisher Scientific) or goat anti-mouse IgG (H+L) (Cat. no. A16066; Invitrogen/Thermo Fisher Scientific) secondary antibody diluted at 1:5,000 in blocking buffer at room temperature for $2 \mathrm{~h}$, followed by ECL (Bio-Rad) detection using a X-ray film or chemiluminescence equipment 


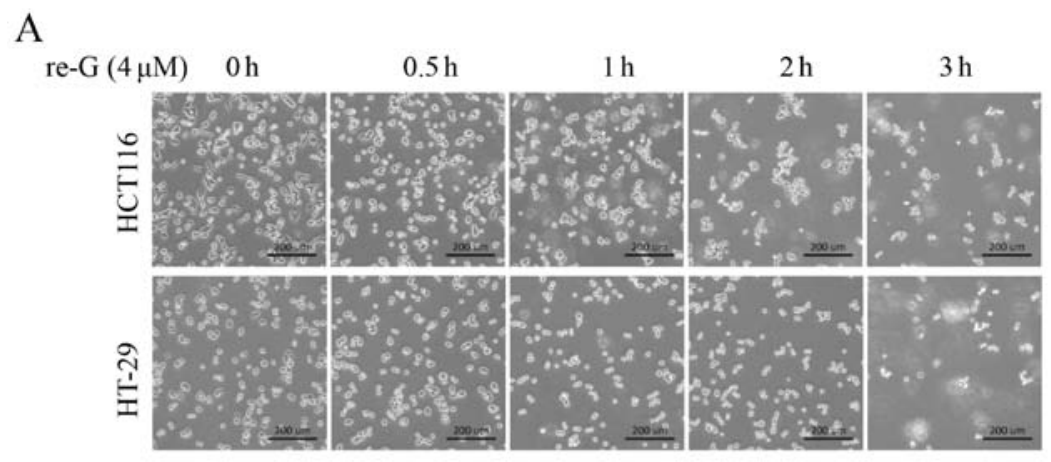

B
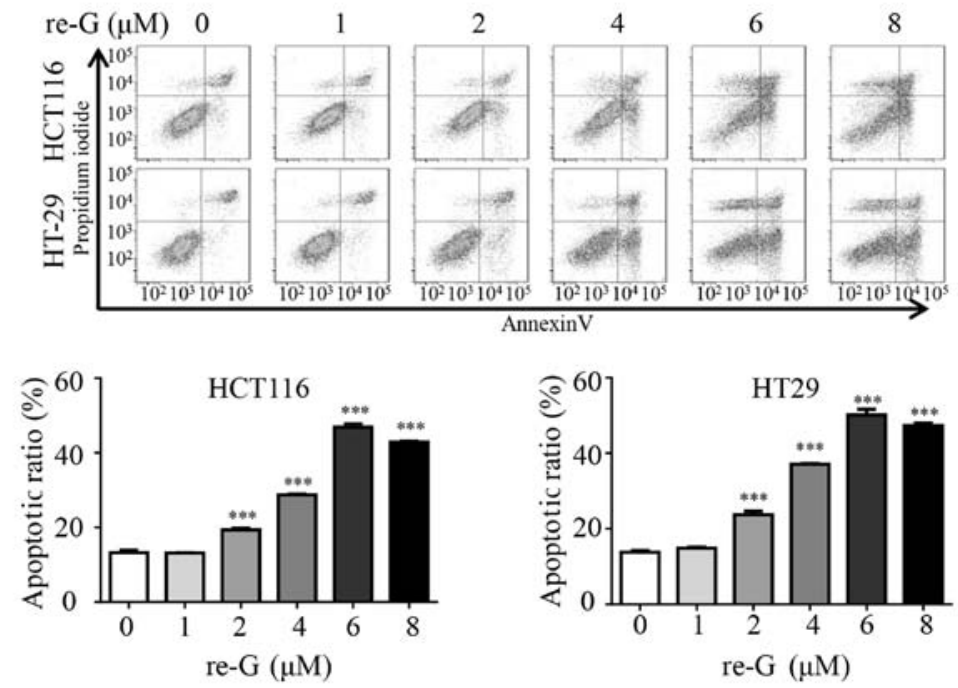

Figure 1. Reduced-gliotoxin induces anoikis in colorectal cancer cells. (A) Reduced-gliotoxin triggered HCT116 and HT-29 cell detachment within 3 h. The HCT116 and HT-29 cells were treated with reduced-gliotoxin at various concentrations and visualized under a phase contrast light microscope. (B) Reduced-gliotoxin induced the death of CRC cells. The HCT116 and HT-29 cells were treated with increasing concentrations of reduced-gliotoxin for $24 \mathrm{~h}$ and apoptosis was detected by Annexin V-FITC/PI double staining with flow cytometry. The apoptotic rate is shown in the bar graphs; all the values are expressed as the means $\pm \mathrm{SD}(\mathrm{n}=3) .{ }^{* * *} \mathrm{P}<0.0001$ vs. untreated cells, as shown by one-way ANOVA with Fisher's LSD test. re-G, reduced-gliotoxin.

(Bio-Rad). After the detection of protein bands, the membranes were stripped and re-probed with anti-GAPDH or anti-ACTB antibodies to confirm equal loading of the samples.

Mitochondrial membrane potential (MMP) assay. MMP in the cells was detected using the MMP assay kit with JC-1 (Beyotime, Shanghai, China). According to the manufacturer's instructions, in brief, the cells were collected after treatment suspended with $5 \mu \mathrm{g} / \mathrm{ml} \mathrm{JC}-1$ in serum-free medium. The cells were then incubated at $37^{\circ} \mathrm{C}$ for $20 \mathrm{~min}$. MMP was detected by flow cytometry (green fluorescence for monomer form, $\mathrm{Ex} / \mathrm{Em}$ : 490/530 nm; red fluorescence for aggregator form, Ex/Em: 525/590 nm).

ROS assay. ROS levels in the cells were monitored using the Reactive Oxygen Species assay kit (Beyotime). As instructed by the manual provided by the manufacturer, the cells were treated with reduced-gliotoxin for the indicated amounts of time, harvested and suspended with $10 \mu \mathrm{M}$ of DCFH-DA in a serum-free medium, and then incubated with dye in $37^{\circ} \mathrm{C}$ for $20 \mathrm{~min}$. The treated cells were washed 3 times with serum-free medium and rinsed in PBS. ROS intensity was measured at an excitation/emission wavelength of $488 / 525 \mathrm{~nm}$ using a flow cytometer (BD Biosciences).
Statistical analysis. All experiments were performed at least 3 times, and the results are expressed as the means \pm SD where applicable. All data were analyzed by a two-tailed unpaired Student's t-test for differences between 2 groups and by one-way ANOVA followed with Fisher's LSD test for multiple comparisons. Statistical analysis was performed using GraphPad Prism software (GraphPad Software, San Diego, CA, USA). A value of $\mathrm{P}<0.05$ was considered to indicate a statistically significant difference.

\section{Results}

Reduced-gliotoxin triggers rapid cell detachment and induces the death of CRC cells. It has previously been reported that gliotoxin triggers rapid cell detachment in mouse embryonic fibroblasts and BEAS-2B human lung bronchial epithelial cells (25). Moreover, we have previously reported that gliotoxin induced the apoptosis of CRC cells (20). Therefore, in this study, we first examined whether reduced-gliotoxin, an analogue of gliotoxin, induces the death of CRC cells via the same mechanism. Indeed, we found that reduced-gliotoxin triggered rapid cell detachment in the HCT116 and HT-29 cells (Fig. 1A). As the detachment of cells can lead to cell death, we further examined the apoptosis-inducing effects of reduced- 
A
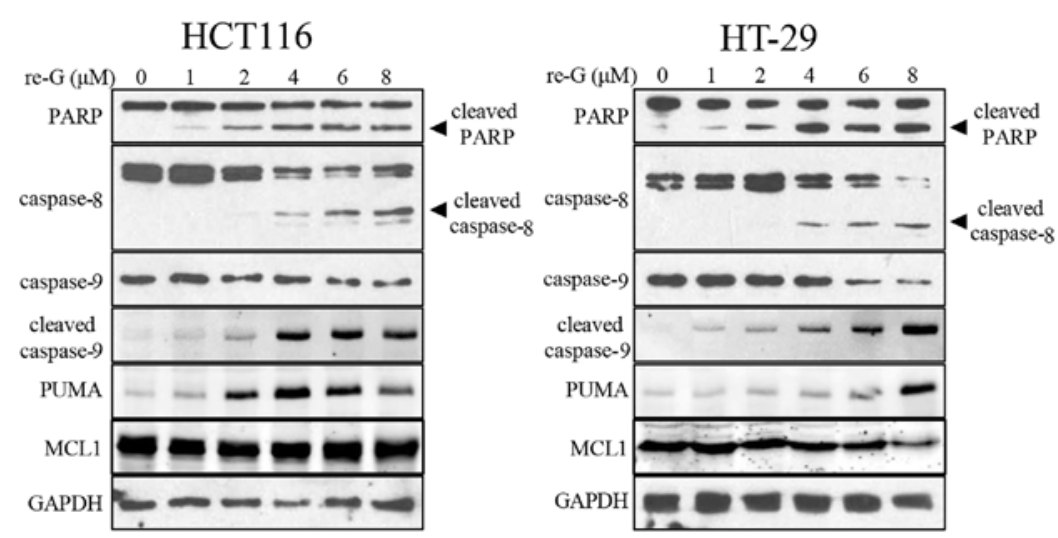

B
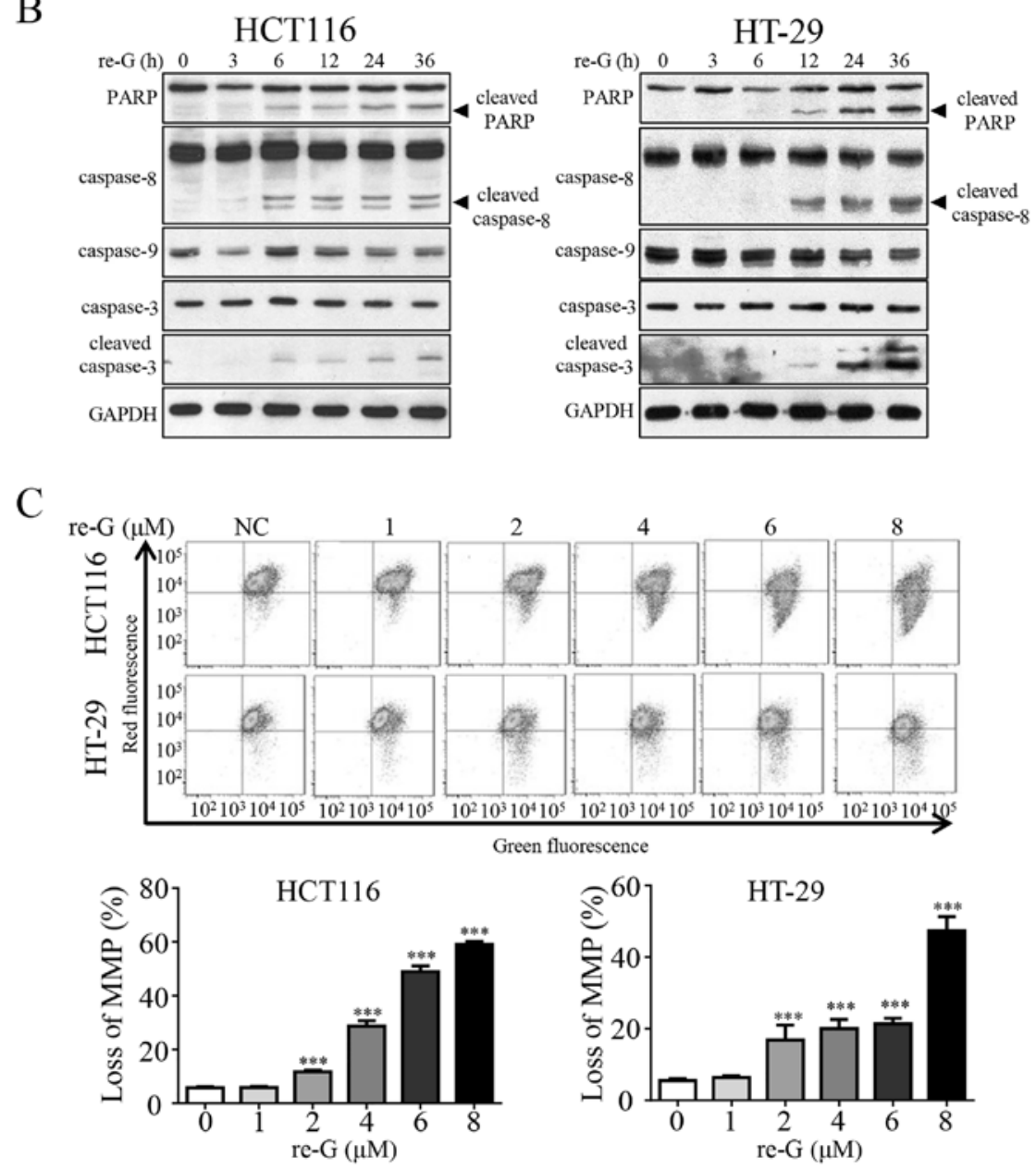

24
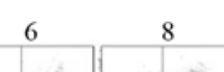
A
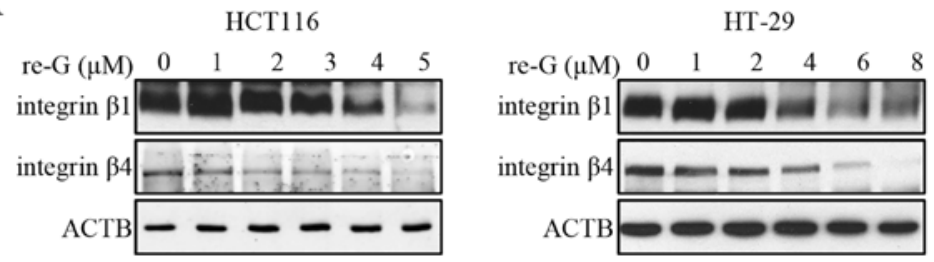

B
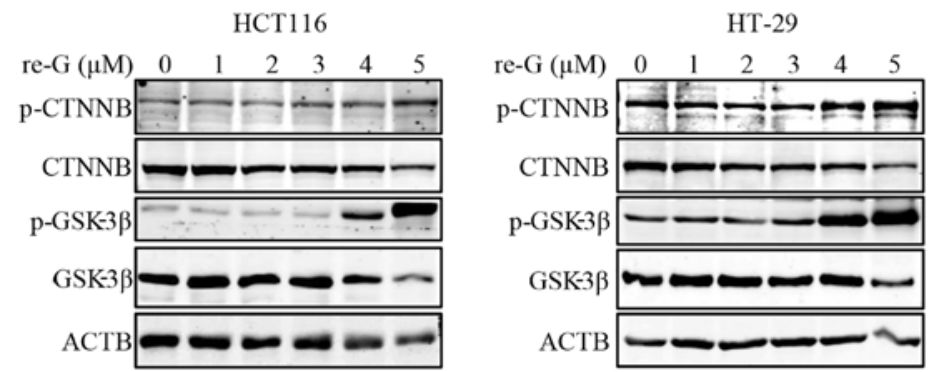

$\mathrm{C}$
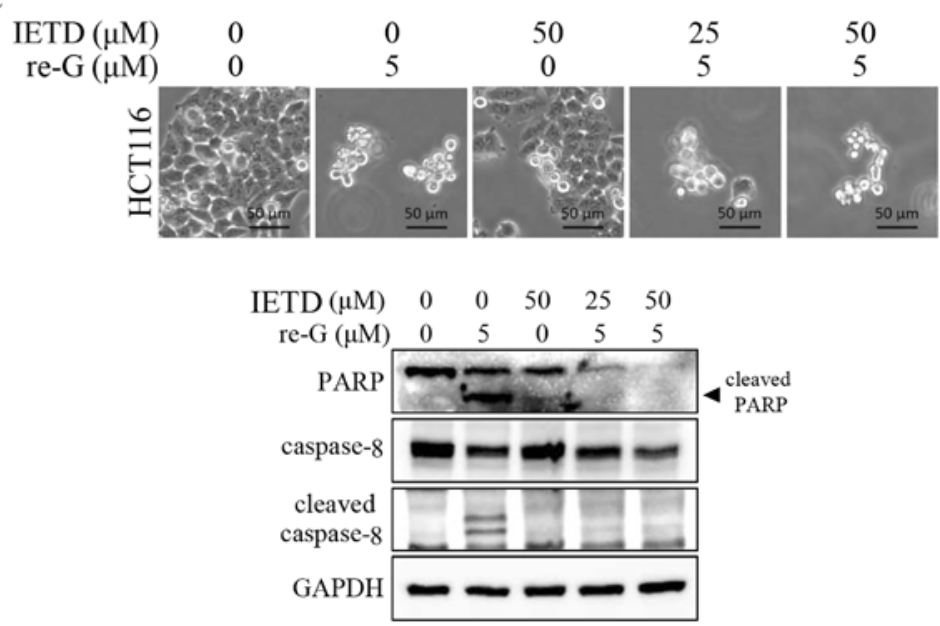

Figure 3. The anoikis induced by reduced-gliotoxin induced is associated with integrin $\beta 1$, integrin $\beta 4$ and $\beta$-catenin degradation, but not is dependent on caspase-8. (A) Reduced-gliotoxin induced integrin $\beta 4$ degradation in the HCT116 and HT-29 cells. The cells were treated with reduced-gliotoxin at the indicated concentrations for $24 \mathrm{~h}$. The protein levels of integrin $\beta 1$ and integrin $\beta 4$ were detected by western blot analyses. ACTB was used as a loading control. (B) $\beta$-catenin degradation induced by reduced-gliotoxin was independent of the activation of GSK-3 $\beta$ in the HCT116 and HT-29 cells. The cells were treated with reduced-gliotoxin at the indicated concentrations for $24 \mathrm{~h}$. The total and phosphorylated protein levels of $\beta$-catenin and GSK-3 $\beta$ were detected by western blot analyses. ACTB was used as a loading control. (C) The inhibition of caspase-8 had no effect on the cell detachment and cell death induced by reduced-gliotoxin. The cells were treated with $5 \mu \mathrm{M}$ reduced-gliotoxin for $24 \mathrm{~h}$, in the absence and presence of the caspase-8 inhibitor, Z-IETD-FMK, at various concentrations. Western blot analyses were performed for the indicated protein. GAPDH was used as a loading control. re-G, reduced-gliotoxin; CTNNB, $\beta$-catenin.

Caspase- 8 is a crucial adaptor in the extrinsic apoptotic pathway and caspase-9 is an important executor in the intrinsic apoptotic pathway (27). Therefore, in this study, we examined the activation of caspase- 8 and caspase- 9 in cells treated with reduced-gliotoxin. The HCT116 and HT-29 cells were treated with reduced-gliotoxin, followed by the measurement of the levels of caspase- 8 , caspase- 9 and PARP by western blot analysis. As shown in Fig. 2A and B, reduced-gliotoxin decreased the total level of caspase- 9 , and increased the levels of cleaved caspase- 8 and PARP in a dose- and time-dependent manner. In addition, reduced-gliotoxin increased the level of PUMA in a dose-dependent manner, whereas the level of MCL1 was not affected. The status of mitochondria has been shown to be deeply involved in cell anoikis (11) and the loss of MMP is considered a hallmark of mitochondrial-associated apoptosis. Therefore, we performed an MMP assay with JC-1 staining and found that JC-1 was gradually spread out as a monomer following treatment with reduced-gliotoxin (Fig. 2C), which indicated that reduced-gliotoxin may disrupt MMP and trigger mitochondrial-associated anoikis. The above-mentioned data suggested that CRC cell apoptosis induced by reduced-gliotoxin might be associated with both the intrinsic and extrinsic apoptotic pathways.

The anoikis induced by reduced-gliotoxin is associated with integrin $\beta 1$, integrin $\beta 4$ and $\beta$-catenin degradation. Given that integrin signaling supports cancer cell metastasis via resisting anoikis $(28,29)$, western blot analysis was performed to evaluate the protein expression of integrin $\beta 1$ and integrin $\beta 4$. As shown in Fig. 3A, treatment of the HCT116 and HT-29 cells with reduced-gliotoxin resulted in a decrease in the protein levels of integrin $\beta 1$ and integrin $\beta 4$. As the loss of $\beta$-catenin has 
A
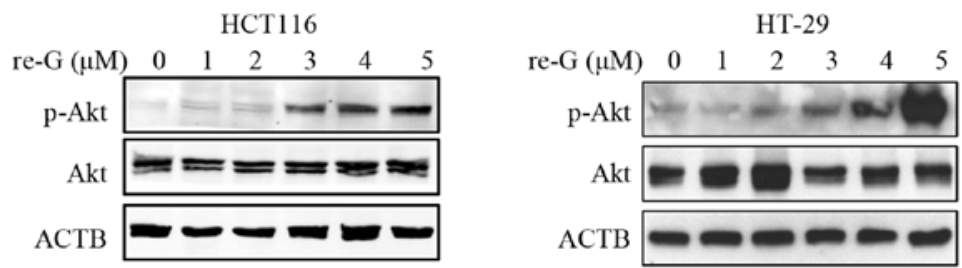

B
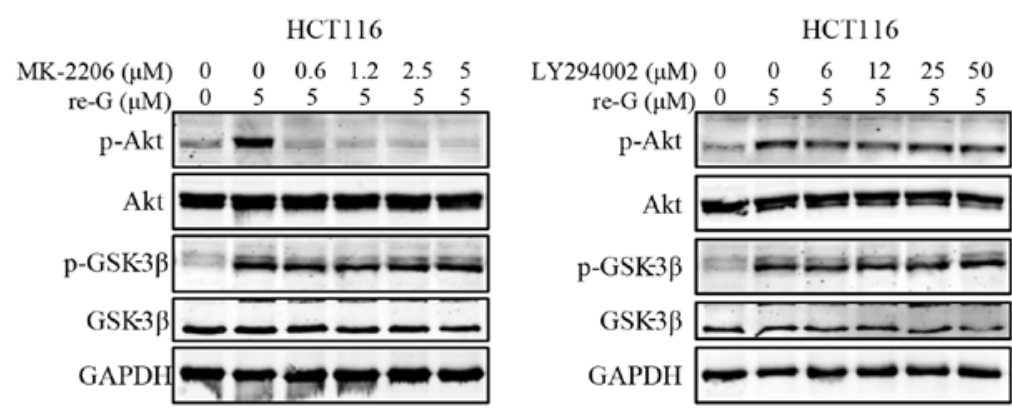

$\mathrm{C}$

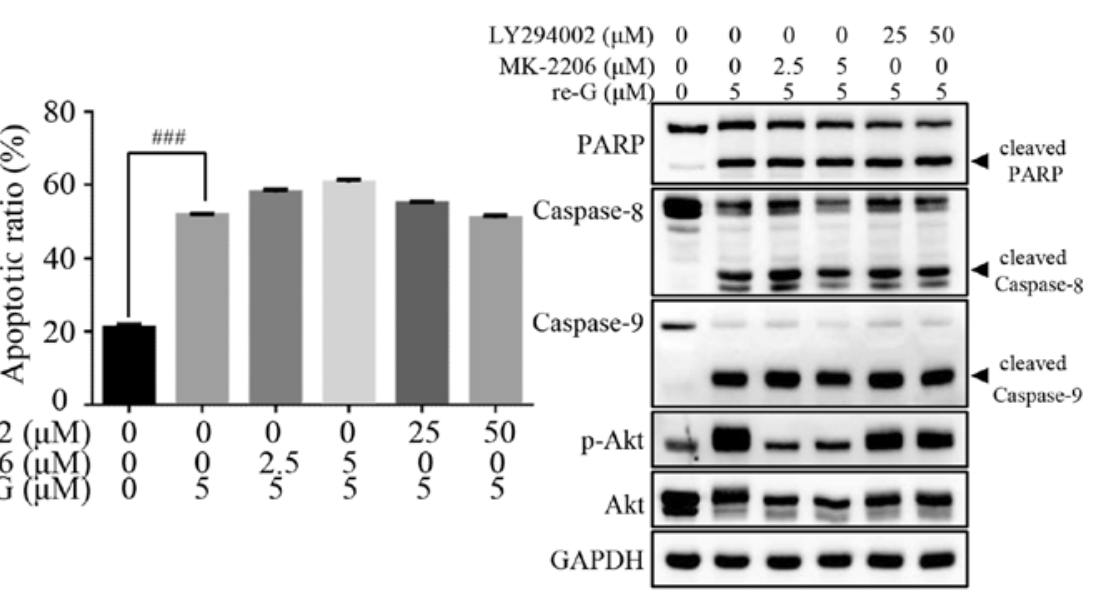

Figure 4. The anoikis induced by reduced-gliotoxin occurs independently of the activation of the Akt/GSK-3 $\beta$ signaling pathway. The HCT116 and HT-29 cells were treated with the indicated concentrations of reduced-gliotoxin for $24 \mathrm{~h}$. Western blot analyses were performed for detecting the total and phosphorylated levels of Akt. (A) Reduced-gliotoxin induced Akt phosphorylation at Ser473 in a dose-dependent manner. (B) The inhibition of PI3K/Akt had no effect on the phosphorylation of GSK-3 $\beta$ induced by reduced-gliotoxin. The cells were treated with $5 \mu \mathrm{M}$ reduced-gliotoxin for $24 \mathrm{~h}$, in the absence and presence of MK-2206 or LY294002 at various concentrations. Western blot analyses were performed for the detection of the indicated proteins. GAPDH was used as a loading control. (C) The inhibition of PI3K/Akt had no effect on the apoptosis induced by reduced-gliotoxin. The cells were treated with $5 \mu \mathrm{M}$ reducedgliotoxin for $24 \mathrm{~h}$, in the absence and presence of MK-2206 or LY294002 at various concentrations. Western blot analyses were performed for the detection of the indicated proteins. GAPDH was used as a loading control. Cell apoptosis was detected by Annexin V-FITC/PI double staining with flow cytometry. The apoptotic rate is shown in the bar graph; all the values are expressed as the means $\pm \mathrm{SD}(\mathrm{n}=3)$. A two-tailed unpaired Student's t-test was applied to examine significant differences in the cells treated with or without reduced-gliotoxin, ${ }^{\# \# \prime} \mathrm{P}<0.001$. re-G, reduced-gliotoxin.

been implicated in triggering anoikis in colon carcinoma (30), we also examined whether reduced-gliotoxin decreases the protein level of $\beta$-catenin by western blot analysis. As shown in Fig. 3B, reduced-gliotoxin decreased $\beta$-catenin expression in a dose-dependent manner. The disengagement of integrin from the ECM leads to the activation of both the extrinsic and intrinsic apoptotic pathways (31). Thus, in order to verify the predominant pathway involved in the execution of the apoptosis of these cells, we used a caspase- 8 inhibitor (Z-IETD-FMK) to block the extrinsic apoptotic pathway. As shown in Fig. 3C, the inhibition of caspase- 8 activity did not reverse cell detachment and death induced by reduced-gliotoxin.

The anoikis induced by reduced-gliotoxin is independent of Akt activation or GSK-3 $\beta$ inactivation. As the phosphorylation of $\beta$-catenin by GSK-3 $\beta$ results in $\beta$-catenin degradation (32), we then examined whether the promotion of $\beta$-catenin degradation induced by reduced-gliotoxin required GSK-3 $\beta$ activity. Of note, reduced-gliotoxin treatment did not modify the expression of pan GSK-3 $\beta$, but induced its phosphorylation on its Ser9 residue, which was shown to trigger its inactivation (Fig. 3B). These results indicate that the degradation of $\beta$-catenin by reduced-gliotoxin occurs independently of GSK-3 $\beta$ activity. During tumorigenesis, PI3K/Akt signaling has been shown to inhibit GSK-3 $\beta$ through phosphorylation at Ser9 (33). Thus, we then examined the phosphorylation of Akt at Ser473, which is strongly dependent upon PI3K activity. As shown in Fig. 4A, treatment of the CRC cells with reduced-gliotoxin increased the level of phosphorylated Akt at Ser473 in a concentration-dependent manner. Furthermore, 


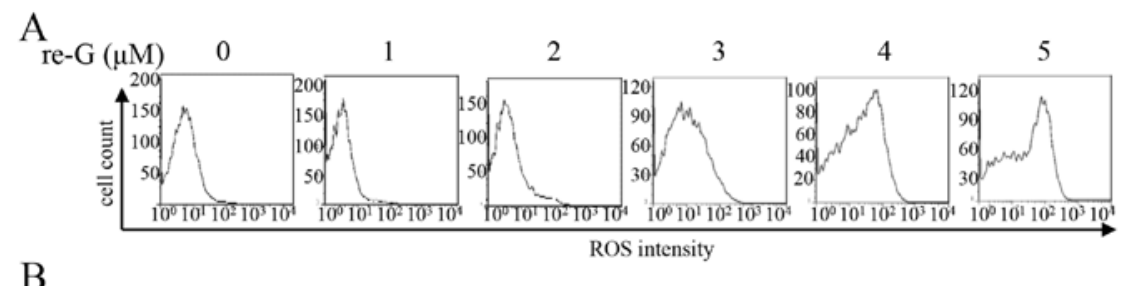

B
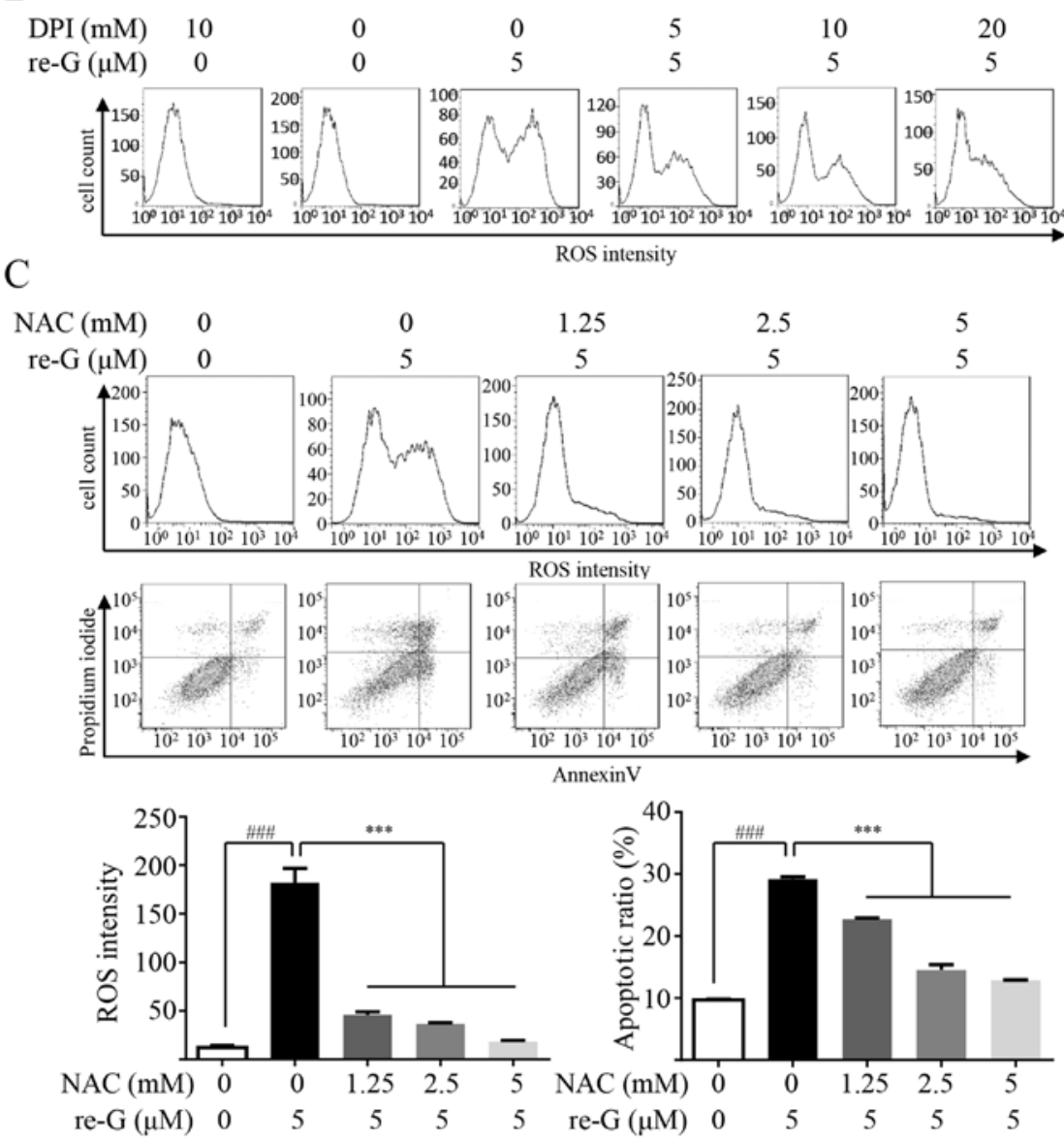

Figure 5. The anoikis induced by reduced-gliotoxin in CRC cells is dependent on reactive oxygen species (ROS) generation. (A) Reduced-gliotoxin induced ROS production in a dose-dependent manner. The cells were treated with the indicated concentrations of reduced-gliotoxin for $24 \mathrm{~h}$, and ROS generation was assessed by 2,7-dichlorodi-hydrofluorescein diacetate (DCFH-DA) staining with flow cytometry. (B) The cells were treated with reduced-gliotoxin $(5 \mu \mathrm{M})$ in the absence and presence of 5,10 and $20 \mathrm{mM}$ of the NOX inhibitor, DPI, for $24 \mathrm{~h}$, and ROS production were then analyzed by flow cytometry (C) The cells were treated with reduced-gliotoxin $(5 \mu \mathrm{M})$ in the absence and presence of 1.25 and $2.5 \mathrm{mM}$ of the ROS scavenger, NAC, for $24 \mathrm{~h}$, and ROS production and apoptotic cell pollutions were then analyzed by flow cytometry. ROS density and the apoptotic rate were shown in the bar graphs; all the values are expressed as the means \pm SD. A two-tailed unpaired Student's t-test was used to determine significant differences between the cells treated with or without reducedgliotoxin, ${ }^{\# \#} \mathrm{P}<0.001$. One-way ANOVA with Fisher's LSD test was used to determine significant differences between the cells treated with NAC and/or reduced-gliotoxin, ${ }^{* * *} \mathrm{P}<0.001$. re-G, reduced-gliotoxin.

we used the PI3K-specific inhibitor, LY294002, and the Akt inhibitor, MK-2206, to examine the role of PI3K/Akt/GSK-3 $\beta$ in the anoikis induced by reduced-gliotoxin. As shown in Fig. 4B, Akt activity was inhibited by treatment with escalating concentrations of MK-2206, whereas the inhibition of GSK-3 $\beta$ induced by reduced-gliotoxin was not affected. Likewise, the activation of Akt induced by reduced-gliotoxin was also not affected by pre-treatment with the PI3K specific inhibitor, LY294002 (Fig. 4B). These findings thus suggest that reduced-gliotoxin triggers the phosphorylation of Akt and that this occurs independently of PI3K activity, while reduced-gliotoxin triggers the phosphorylation of GSK-3 $\beta$ and that this occurs independently of the PI3K/Akt pathway. We also examined the apoptosis-promoting effects of reducedgliotoxin by flow cytometric analysis and western blot analysis in the presence or absence of PI3K/Akt inhibitors. As shown in Fig. 4C, PI3K/Akt inhibition did not affect the cell detachment and death induced by reduced-gliotoxin.

Excessive ROS production is the upstream regulator of the anoikis induced by reduced-gliotoxin. Gliotoxin has been found to increase ROS generation and induce oxidative stress in mouse fibroblasts (34), human hepatic stellate cells (35) and cultured macrophages (36). ROS generation is associated with the mitochondria, and as shown above, reduced-gliotoxin triggered the loss of MMP (Fig. 2B). Therefore, we examined the intracellular ROS levels by flow cytometry in the cells with or without reduced-gliotoxin treatment. As shown in Fig. 5A, reduced-gliotoxin increased ROS production in a dose-dependent manner. The two main sources of ROS are the 
A

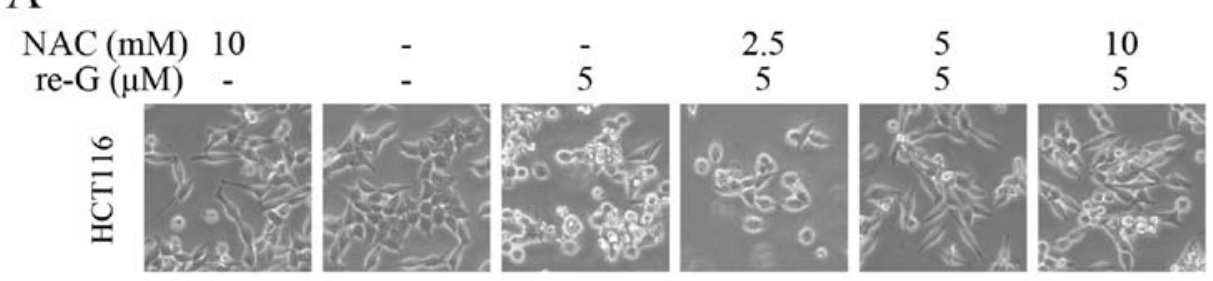

B

$\mathrm{C}$
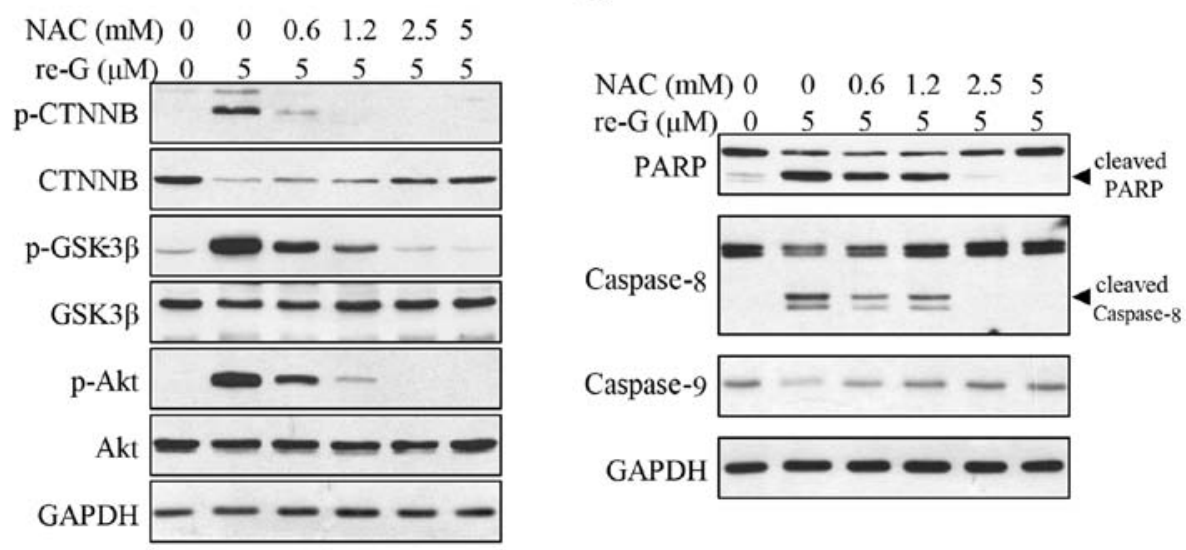

Figure 6. Reactive oxygen species (ROS) generation is involved in cell detachment, the activation of multiple signaling pathways, and cell anoikis induced by reduced-gliotoxin. The cells were incubated with or without various concentrations of NAC for $2 \mathrm{~h}$ prior to exposure to reduced-gliotoxin $(5 \mu \mathrm{M})$ for $24 \mathrm{~h}$. (A) The cell status was visualized under a phase contrast light microscope. (B) Western blot analyses were performed for the detection of the activation of Akt/ GSK-3 $\beta / \beta$-catenin signaling pathways, and GAPDH was used as a loading control. (C) Western blot analyses were performed for the detection of the status of apoptotic markers. GAPDH was used as a loading control.

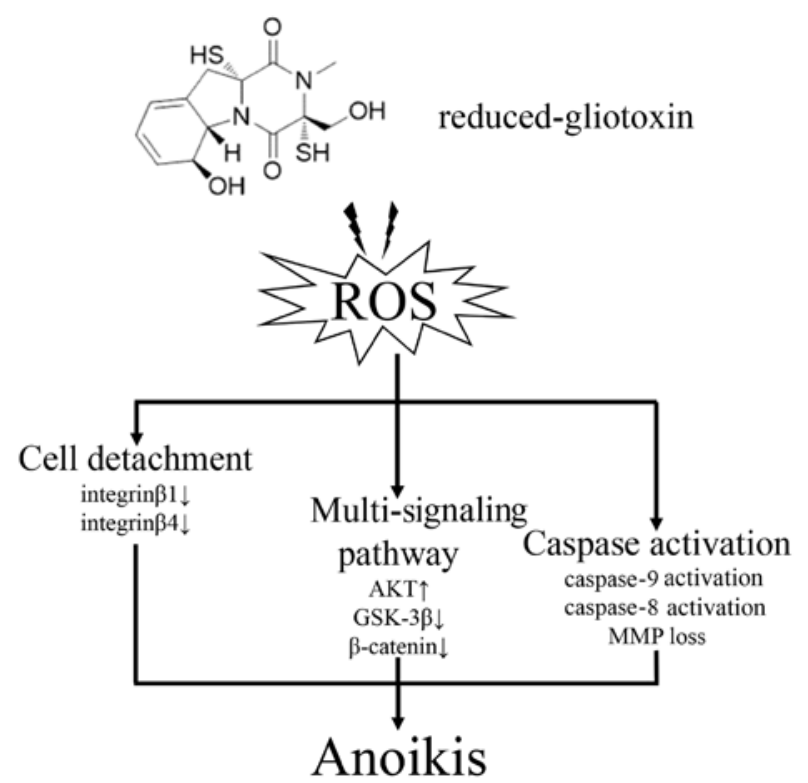

Figure 7. Schematic illustration of the underlying mechanisms responsible for the anticancer effects of reduced-gliotoxin.

mitochondria and NADPH oxidase (NOX). In order to verify the main source of ROS generation induced by treatment with reduced-gliotoxin, we used DPI to block NOX activity. As it shown in Fig. 5B, DPI reduced most of the ROS generation. To identify the role of ROS in the anoikis induced by reduced-gliotoxin, NAC, as a ROS scavenger, was used. Of note, it was found that NAC almost completely abolished cell anoikis induced by reduced-gliotoxin (Fig. 5C). We further examined the effects of NAC on cell detachment, and multisignaling pathway and caspase cascade activation induced by reduced-gliotoxin. As shown in Fig. 6A, the cell detachment induced by reduced-gliotoxin was attenuated by pre-treatment with NAC. In addition, $\beta$-catenin degradation, Akt activation and GSK-3 $\beta$ inhibition induced by reduced-gliotoxin-induced were all reversed (Fig. 6B). Additionally, the decrease in the levels of PARP, caspase- 8 cleavage and pro-caspase- 9 induced by reduced-gliotoxin were all reversed by NAC pre-treatment (Fig. 6C). These results suggest that ROS induction mediates the apoptotic pathways activated by reduced-gliotoxin and that ROS are a critical upstream regulator of the anoikis induced by reduced-gliotoxin.

\section{Discussion}

There is evidence to suggest that gliotoxin is a promising anticancer reagent that induces cytotoxic effects on cancer cells (21-23). Previously, we observed that reduced- exerted the most potent anticancer effects compared to other gliotoxin analogues (14). In this study, we examined the potency of reduced-gliotoxin against $\mathrm{CRC}$ cells and the underlying mechanisms. We demonstrated that reduced-gliotoxin triggered rapid cell detachment and induced the apoptosis of CRC cells. Mechanistically, our data indicated that the anoikis induced by reduced-gliotoxin induced is associated with the disruption of integrin-related cell detachment and multiple signaling pathways. Furthermore, reduced-gliotoxin induced ROS production, resulting in the activation of both endogenous and exogenous apoptotic pathways and eventually resulting in the apoptosis of CRC cells. The blockade of ROS generation by 
NAC completely reversed several reduced-gliotoxin-induced protein kinase signaling pathways and cell anoikis (Fig. 7). Taken together, these studies suggest that reduced-gliotoxin may be a potential candidate for the treatment of CRC.

Anoikis is defined as apoptosis that is induced by inadequate or inappropriate cell matrix interaction (11). Anoikis plays an important role in tissue homeostasis, disease development and cancer metastasis (5). Resistance to anoikis characterizes the ability of cancer cells to circumvent barriers to migration and invasion, thereby promoting metastatic progression (31). Therefore, a novel method that can enhance or induce anoikis to disrupt the settling of metastatic cancer cells at a distal site is an attractive strategy. In this study, we demonstrated that reducedgliotoxin induced cell death by inducing anoikis (Fig. 1). Anoikis either disrupts mitochondrial homeostasis or triggers cell surface death receptors; hence, it is involved in both the intrinsic and extrinsic apoptotic pathways (11). In this study, the anoikis induced by reduced-gliotoxin was characterized by the activation of caspase-8, caspase-9 and PARP cleavage, suggesting that the intrinsic apoptotic pathway mainly mediates this event.

The induction of anoikis is caused by an absence of adhesive interaction between epithelial cells and their surrounding ECM. Integrins involved in the cell-ECM and cell-cell communication play an important role in regulating anoikis and metastasis (37). The overexpression of integrin $\beta 1$ and integrin $\beta 4$, which promote cell invasion and metastasis, are associated with a clinically aggressive phenotype of different types of cancer, including CRC (38-40). The inhibition of cell surface integrins is able to influence the first step of anoikis. It has been suggested this may prove to be an attractive target for CRC therapy (41). In this context, in this study, we demonstrated that the anoikis induced by reduced-gliotoxin might be associated with integrin $\beta 1$ and integrin $\beta 4$ degradation (Fig. 3). Previously, we reported that gliotoxin blocked the Wnt/ $\beta$-catenin signaling pathway by inducing the degradation of $\beta$-catenin in CRC cells. A similar result was observed: Reduced-gliotoxin induced $\beta$-catenin phosphorylation and decreased the protein expression levels. $\beta$-catenin is a major oncoprotein that is often activated in CRC and promotes tumor progression. Our data indicated that reduced-gliotoxin induced CRC cell anoikis and this was not only associated with the degradation of proteins interacting with ECM, but also with adherens junction proteins. Furthermore, we demonstrated that the degradation of $\beta$-catenin was accompanied by Akt activation and GSK- $3 \beta$ inactivation.

Reduced-gliotoxin can be oxidized to gliotoxin, thus releasing ROS, and it can also form mixed disulfides by thiol groups with proteins or antioxidants, such as glutathione (42). Therefore, it is not surprising to observe that ROS was produced by CRC cells treated with reduced-gliotoxin, whereas pre-treatment with NAC blocked ROS production and cell anoikis. In tumorigenesis, GSK-3 $\beta$ is the most important kinase for the abnormal phosphorylation of $\beta$-catenin, and the inactivation of GSK-3 $\beta$ promotes the accumulation of $\beta$-catenin. In this study, reduced-gliotoxin triggered the activation of Akt and the inactivation of GSK-3 $\beta$. Moreover, the activation of Akt and the inactivation of GSK-3 $\beta$ were abrogated by pre-treatment with NAC. NAC inhibited the phosphorylation of Akt; it subsequently inactivated GSK-3 $\beta$ by phosphorylating at Ser9 as usual. However, inhibiting the activation of Akt with LY294002 or MK-2206 did not attenuate the inactivation of GSK-3 $\beta$. Thus, ROS may act independently of Akt activation to mediate reduced-gliotoxin, which inactivates GSK-3 $\beta$ activity. Based on these findings, it is likely that ROS are crucial factors in the induction of anoikis and act as upstream signaling molecules in initiating cell anoikis.

\section{Acknowledgements}

The authors would like to thank Rose-Ann Thomas for English proofreading the manuscript.

\section{Funding}

This study was supported by National Natural Science Foundation of China (81672413 and 81402418); Doctoral Funds of Ministry of Education of China (2016M592583); Guangdong Provincial Department of Science and Technology (2014B020212016); Guangdong Natural Science Funds (2015A030310096); Guangzhou Science Technology and Innovation Commission (2016201604030007; 2016201604030003); Overseas Excellent Professor Project, Ministry of Education, China; Japan Ministry of Education, Culture, Sports, Science and Technology (MEXT) for Program of Japan Initiative for Global Research Network on Infectious Diseases (J-GRID).

\section{Availability of data and materials}

All data generated or analyzed during this study are included in this published article.

\section{Authors' contributions}

JC, QL, LH, CW, ML, ZZ, FW and LH performed the cell and molecular biology experiments; WL contributed to the marine natural productions; JC and QL wrote the manuscript; AI revised the manuscript and provided professional advice during the whole research process. HL and XY designed the experiments and analyzed the results. All authors read and approved the final manuscript.

\section{Ethics approval and consent to participate}

Not applicable.

\section{Consent for publication}

Not applicable.

\section{Competing interests}

The authors declare that they have no competing interests.

\section{References}

1. Siegel RL, Miller KD and Jemal A: Cancer statistics, 2016. CA Cancer J Clin 66: 7-30, 2016.

2. Chen W, Zheng R, Baade PD, Zhang S, Zeng H, Bray F, Jemal A Yu XQ and He J: Cancer statistics in China, 2015. CA Cancer J Clin 66: 115-132, 2016. 
3. Siegel RL, Miller KD, Fedewa SA, Ahnen DJ, Meester RGS, Barzi A and Jemal A: Colorectal cancer statistics, 2017. CA Cancer J Clin 67: 177-193, 2017.

4. März L and Piso P: Treatment of peritoneal metastases from colorectal cancer. Gastroenterol Rep (Oxf) 3: 298-302, 2015.

5. Gilmore AP: Anoikis. Cell Death Differ 12 (Suppl 2): 1473-1477, 2005.

6. Chiarugi P and Giannoni E: Anoikis: A necessary death program for anchorage-dependent cells. Biochem Pharmacol 76: 1352-1364, 2008.

7. Grossmann J: Molecular mechanisms of 'detachment-induced apoptosis - Anoikis'. Apoptosis 7: 247-260, 2002.

8. Simpson CD, Anyiwe K and Schimmer AD: Anoikis resistance and tumor metastasis. Cancer Lett 272: 177-185, 2008.

9. Nagaprashantha LD, Vatsyayan R, Lelsani PC, Awasthi S and Singhal SS: The sensors and regulators of cell-matrix surveillance in anoikis resistance of tumors. Int J Cancer 128: 743-752, 2011.

10. Shanmugathasan $\mathrm{M}$ and Jothy S: Apoptosis, anoikis and their relevance to the pathobiology of colon cancer. Pathol Int 50: 273-279, 2000

11. Frisch SM and Screaton RA: Anoikis mechanisms. Curr Opin Cell Biol 13: 555-562, 2001.

12. Cragg GM and Newman DJ: Natural products: A continuing source of novel drug leads. Biochim Biophys Acta 1830: 3670-3695, 2013

13. Saleem M, Ali MS, Hussain S, Jabbar A, Ashraf M and Lee YS Marine natural products of fungal origin. Nat Prod Rep 24: 1142$1152,2007$.

14. Liang WL, Le X, Li HJ, Yang XL, Chen JX, Xu J, Liu HL, Wang LY, Wang KT, Hu KC, et al: Exploring the chemodiversity and biological activities of the secondary metabolites from the marine fungus Neosartorya pseudofischeri. Mar Drugs 12: 5657-5676, 2014

15. Bugni TS and Ireland CM: Marine-derived fungi: A chemically and biologically diverse group of microorganisms. Nat Prod Rep 21: 143-163, 2004

16. Lan WJ, Fu SJ, Xu MY, Liang WL, Lam CK, Zhong GH, Xu J, Yang DP and Li HJ: Five new cytotoxic metabolites from the marine fungus Neosartorya pseudofischeri. Mar Drugs 14: 18, 2016.

17. Yan DF, Lan WJ, Wang KT, Huang L, Jiang CW and Li HJ: Two chlorinated benzofuran derivatives from the marine fungus Pseudallescheria boydii. Nat Prod Commun 10: 621-622, 2015.

18. Yamada A, Kataoka T and Nagai K: The fungal metabolite gliotoxin: Immunosuppressive activity on CTL-mediated cytotoxicity. Immunol Lett 71: 27-32, 2000

19. Sun Y, Takada K, Takemoto Y, Yoshida M, Nogi Y, Okada S and Matsunaga S: Gliotoxin analogues from a marine-derived fungus, Penicillium sp., and their cytotoxic and histone methyltransferase inhibitory activities. J Nat Prod 75: 111-114, 2012.

20. Chen J, Wang C, Lan W, Huang C, Lin M, Wang Z, Liang W, Iwamoto A, Yang $\mathrm{X}$ and Liu H: Gliotoxin inhibits proliferation and induces apoptosis in colorectal cancer cells. Mar Drugs 13: 6259-6273, 2015

21. Nguyen VT, Lee JS, Qian ZJ, Li YX, Kim KN, Heo SJ, Jeon YJ, Park WS, Choi IW, Je JY, et al: Gliotoxin isolated from marine fungus Aspergillus sp. induces apoptosis of human cervical cancer and chondrosarcoma cells. Mar Drugs 12: 69-87, 2013.

22. Hubmann R, Hilgarth M, Schnabl S, Ponath E, Reiter M, Demirtas D, Sieghart W, Valent P, Zielinski C, Jäger U, et al: Gliotoxin is a potent NOTCH2 transactivation inhibitor and efficiently induces apoptosis in chronic lymphocytic leukaemia (CLL) cells. Br J Haematol 160: 618-629, 2013.

23. Pan XQ and Harday J: Electromicroscopic observations on gliotoxin-induced apoptosis of cancer cells in culture and human cancer xenografts in transplanted SCID mice. In Vivo 21: 259-265, 2007.
24. Burnette WN: 'Western blotting': Electrophoretic transfer of proteins from sodium dodecyl sulfate - polyacrylamide gels to unmodified nitrocellulose and radiographic detection with antibody and radioiodinated protein A. Anal Biochem 112: 195-203, 1981.

25. Geissler A, Haun F, Frank DO, Wieland K, Simon MM, Idzko M, Davis RJ, Maurer U and Borner C: Apoptosis induced by the fungal pathogen gliotoxin requires a triple phosphorylation of Bim by JNK. Cell Death Differ 20: 1317-1329, 2013.

26. Maamer-Azzabi A, Ndozangue-Touriguine $O$ and Bréard J: Metastatic SW620 colon cancer cells are primed for death when detached and can be sensitized to anoikis by the BH3-mimetic ABT-737. Cell Death Dis 4: e801, 2013.

27. Fulda $S$ and Debatin KM: Extrinsic versus intrinsic apoptosis pathways in anticancer chemotherapy. Oncogene 25: 4798-4811, 2006.

28. Ata R and Antonescu CN: Integrins and cell metabolism: An intimate relationship impacting cancer. Int J Mol Sci 18: 18, 2017.

29. Alanko J, Mai A, Jacquemet G, Schauer K, Kaukonen R, Saari M, Goud B and Ivaska J: Integrin endosomal signalling suppresses anoikis. Nat Cell Biol 17: 1412-1421, 2015.

30. Yoo BH, Masson O, Li Y, Khan IA, Gowda PS and Rosen KV: Anoikis of colon carcinoma cells triggered by $\beta$-catenin loss can be enhanced by tumor necrosis factor receptor 1 antagonists. Oncogene 34: 4939-4951, 2015

31. Paoli P, Giannoni E and Chiarugi P: Anoikis molecular pathways and its role in cancer progression. Biochim Biophys Acta 1833: 3481-3498, 2013

32. Liu C, Li Y, Semenov M, Han C, Baeg GH, Tan Y, Zhang Z, Lin X and He X: Control of beta-catenin phosphorylation/degradation by a dual-kinase mechanism. Cell 108: 837-847, 2002.

33. Luo J: Glycogen synthase kinase 3beta (GSK3beta) in tumorigenesis and cancer chemotherapy. Cancer Lett 273: 194-200, 2009.

34. Pardo J, Urban C, Galvez EM, Ekert PG, Müller U, Kwon-Chung J, Lobigs M, Müllbacher A, Wallich R, Borner C, et al: The mitochondrial protein Bak is pivotal for gliotoxin-induced apoptosis and a critical host factor of Aspergillus fumigatus virulence in mice. J Cell Biol 174: 509-519, 2006.

35. Kweon YO, Paik YH, Schnabl B, Qian T, Lemasters JJ and Brenner DA: Gliotoxin-mediated apoptosis of activated human hepatic stellate cells. J Hepatol 39: 38-46, 2003.

36. Suen YK, Fung KP, Lee CY and Kong SK: Gliotoxin induces apoptosis in cultured macrophages via production of reactive oxygen species and cytochrome c release without mitochondrial depolarization. Free Radic Res 35: 1-10, 2001.

37. Ruoslahti E and Reed JC: Anchorage dependence, integrins, and apoptosis. Cell 77: 477-478, 1994.

38. Beaulieu JF: Integrin $\alpha 6 \beta 4$ in colorectal cancer. World J Gastrointest Pathophysiol 1: 3-11, 2010.

39. Scartozzi M, Giampieri R, Loretelli C, Mandolesi A, del Prete M, Biagetti S, Alfonsi S, Faloppi L, Bianconi M, Bittoni A, et al: Role of $\beta 4$ integrin in HER-3-negative, K-RAS wild-type metastatic colorectal tumors receiving cetuximab. Future Oncol 9: 1207-1214, 2013.

40. Fujita S, Watanabe M, Kubota T, Teramoto T and Kitajima M: Alteration of expression in integrin beta 1-subunit correlates with invasion and metastasis in colorectal cancer. Cancer Lett 91: 145-149, 1995.

41. Pelillo C, Mollica H, Eble JA, Grosche J, Herzog L, Codan B, Sava $\mathrm{G}$ and Bergamo A: Inhibition of adhesion, migration and of $\alpha 5 \beta 1$ integrin in the HCT-116 colorectal cancer cells treated with the ruthenium drug NAMI-A. J Inorg Biochem 160: 225-235, 2016.

42. Scharf DH, Brakhage AA and Mukherjee PK: Gliotoxin - bane or boon? Environ Microbiol 18: 1096-1109, 2016. 